\title{
Curative-intent surgery for pancreatic tumors: a review of 3,386 procedures from the Brazilian National Health System
}

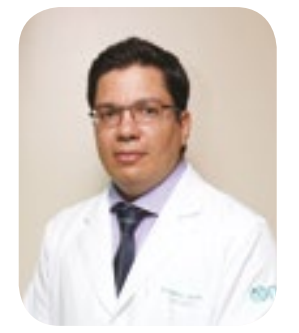

\author{
Marco Antonio O. Lessa', Benjamin Haaland², Gilberto de Lima \\ Lopes $^{3}$, Lucas Vieira dos Santos ${ }^{4}$
}

\section{INTRODUÇÃO}

O tratamento do câncer de pâncreas é um desafio para oncologistas clínicos e cirurgiões, tendo em vista a sua agressividade, uma vez que $95 \%$ dos pacientes diagnosticados irão morrer em 1 ano. ${ }^{1}$

O tipo histológico mais comum é o adenocarcinoma, representando $85 \%$ dos casos. ${ }^{2} \mathrm{E}$ a melhor opção terapêutica se inicia preferencialmente por cirurgias complexas e mórbidas, as quais são duodenopancreatectomia (DPT) ou pancreatectomia parcial (PTP), ambas associadas à linfadenectomia, porém esta abordagem curativa só é possível em cerca de $20 \%$ dos pacientes diagnosticados. ${ }^{3}$

A Constituição Federal Brasileira estabelece que "a saúde é direito de todos e dever do estado". ${ }^{4}$ Todavia, o SUS não atinge seus objetivos de universalidade e integralidade, e as disparidades regionais são algumas das consequências deste sistema ineficiente. Esta disparidade do acesso à saúde é muito mais evidente quando cruzamos com a taxa de desenvolvimento econômico. ${ }^{5}$ Sendo assim, este estudo se propõe a avaliar o impacto das desigualdades socioeconômicas brasileiras no tratamento cirúrgico, com intuito curativo do câncer de pâncreas (CP).

\section{METODOLOGIA}

Neste estudo foi realizada a análise dos dados sobre procedimentos cirúrgicos com intenção curativa para CP no período de janeiro de 2008 a julho de 2014, nos vinte e sete estados da Federação, incluindo o Distrito Federal. Foram obtidos pelo sistema DATASUS os dados referentes ao número de procedimentos, custos, tempo de duração da internação, número de óbitos perioperatórios e mortes relacionadas a câncer de pâncreas. ${ }^{6}$ Do Instituto Brasileiro de Geografia e Estatística (IBGE) foram coletados os números referentes ao tamanho da população e o Produto Interno Bruto (PIB) per capta de cada estado da Federação. ${ }^{7}$
As variáveis de mortalidade por câncer de pâncreas, taxa de mortalidade relacionada ao procedimento cirúrgico, custos, duração da internação foram analisadas as suas correlações com o número de procedimentos, PIB per capta e população através da correlação de Spearman, sendo necessário um $p$ menor ou igual a 0,05 para se determinar significância estatística.

\section{RESULTADOS}

No Brasil, 3.386 procedimentos cirúrgicos para tratamento com intenção curativa para neoplasia pancreática foram realizados no período estudado. No gráfico 1 observa-se que $51 \%$ destes procedimentos foram realizados na Região Sudeste, sendo o estado de São Paulo responsável por $32,7 \%$ de todos os procedimentos realizados no Brasil. O gráfico 2 evidencia a mortalidade secundária a câncer de pâncreas por 100 mil pessoas-ano, apresentando valores maiores nas Regiões Sudeste $(4,64)$ e Sul $(5,98)$. Nas demais: Centro-Oeste, Nordeste e Norte apresentam os respectivos valores: 2,96, 2,42 e 1,49/100 mil pessoas-ano.

A taxa de mortalidade específica por câncer de pâncreas tende a crescer quanto maior for o número de procedimentos cirúrgicos, PIB per capita e população, como se verifica na tabela 1. Porém, quando realizada a análise multivariada (tabela 1 ), verifica-se que, mantendo o número de procedimentos e a população fixos, a taxa de mortalidade cresceria com o PIB per capita $0,72(0,06-1,38 ; p=0,033)$.

A taxa de mortalidade relacionada à DPT e PTP foi de $14,56 \%$. No gráfico 3 verificamos que a Região Norte se destaca por ter a maior taxa de mortalidade (25\%), porém dois estados se destacam negativamente, com taxas de 33\% no Pará e 38\% em Sergipe.

$O$ custo total referente à realização dos procedimentos no Brasil no período estudado foi de 21.528.306,78 reais. A Região Sudeste e, em especial, o estado de São 
Paulo apresentaram os maiores gastos, respectivamente $11.290 .016,88$ e $7.651 .514,49$ de reais. Esses custos aumentam quanto maior o número de procedimentos realizados e a populaçăo, porem o PIB per capita não interfere no custo, como e possivel veriticar na tabela 3. A análise multivariada demonstra que os custos irão crescer, caso haja um número maior de procedimentos, mas irăo decrescer, caso ocorra um aunento da população residente no estado.

O tempo de internação médio relacionado ao procedimento cirúrgico no Brasil foi de 16,9 dias. O gráfico 4 mostra que a Região Nordeste apresentou menor tempo, com 14,9 dias, e o Centro-Oeste teve o maio tempo, com 18,7 dias. No entanto, verificamos que o tempo de internação não tem associação direta com o número de procedimentos, PIB per
da população, conforme tabela 2.

\section{DISCUSSÃO}

objetivo deste estudo foi tentar avaliar, de forma pioneira, se as desigualdades socioeconômicas do Brasil interfeririam nos resultados sobre o manejo curativo do CP. Sendo assim, foram analisados os números de procedimentos realizados, as taxas de mortalidade por $\mathrm{CP}$ e relacionada ao procedimento, custos, duração da internação, PIB per capta e tamanho da população.

O Brasil é um país de dimensões continentais, com IDH intermediário, um sistema de saúde público universal, porém apenas $75 \%$ da população faz uso exclusivo deste serviço, e enormes disparidades regionais. A população do país está majoritariamente localizada nos estados do Centro-Sul e são estas mesmas regiões que apresentam os melhores indices econômicos e de desenvolvimento humano. ${ }^{7}$ Este estudo permitiu examinar o impacto dessas disparidades nos dados brutos de eficácia do tratamento inicial ao $\mathrm{CP}$.

tratamento com intenção curativa para pacientes com CP é desafiador per si. Diversos fatores estão associados as taxas de morbimortalidade, como o estado nutricional, o estáglo da doença, a presença de comorbidades e o status de desempenho. ${ }^{, 9}$ Como neste estudo lidamos com dados agregados, desta forma dados sobre a disponibilidade de tratamentos oncologicos adjuvantes não puderam ser analisados.

Os resultados obtidos sugerem que o acesso à saúde e os indicadores de eficiência do SUS são desiguais entre as diversas unidades da federação. $O$ número de procedimentos cirúrgicos e a mortalidade por CP é maior nos estados do Centro-Sul, notadamente no estado de São Paulo, o que não se justifica pelas estimativas de incidência, e sim pela ocorrência de migração inter-regional proveniente de regiões com menor assistência à saúde, os quais, por lei, deveriam ser oferecidos ate mesmo em estados mais pobres. ${ }^{4}$ A associação da taxa de mortalidade específica por CP com o número de procedimentos realizados e PIB per capita pode ser o reflexo de maior incidência nestas regiões mais desenvolvidas, como também pela migração regional. Nos grandes centros internacionais verificamos que as taxas de mortalidade relacionada à cirurgia com intuito curativo para câncer de pâncreas são menores de $5 \%$. " $A$ mortalidade perioperatória é de $14,55 \%$ no Brasil, porem o pior resultado é de Sergipe, onde a taxa e de $38 \%$. Infelizmente, apesar da discrepancia dos dados, não foi possivel determinar se esta mortalidade perioperatória estaria relacionada ao número de procedimentos, PIB per capita ou tamanho da população. A ausência de registros relacionados à realização de procedimentos com intenção curativa em Roraima no período de tempo estudado demonstra a precariedade do atendimento à saúde neste estado, bem como abre questionamentos sobre a qualidade dos dados gerados pelas instituições e agências governamentais.

O tempo de internação após o procedimento cirúrgico médio no Brasil foi de 16,9 dias, o que se mostrou muito superior ao encontrado em grandes centros internacionais, onde a média é de 7 dias. ${ }^{8}$ Esta discrepância nos resultados indicam que regiões subdesenvolvidas podem apresentar uma menor qualidade no cuidado dos pacientes com CP. E o tempo de internamento é variável mesmo no Brasil, sendo maior em regiões com menor PIB per capta, a exemplo de Rondônia.

Os gastos referentes ao tratamento dos pacientes com câncer de pâncreas mantiveram-se em ressonâncla com os resultados anteilores, que mostraram o Sudeste como polo no tratamento desta patologia. $\mathrm{O}$ custo relativo, entretanto, se reduz nos estados mais populosos, sugerindo uma economia em escala. ${ }^{0}$

Este estudo tem as limitações inerentes à sua natureza retrospectiva, bem como a utilização de dados agregados (e não individuais). A qualidade dos dados limita-se ainda pela eficiência das agências governamentais em coletar informações que sejam confiáveis e abrangentes. Todavia, o grande numero de procedimentos analisados (mais de 3,300 ) em um pais com dimensões continentais e mais de 200 milhões de habitantes parece tornar os dados robustos o suficiente para aumentar a confiabilidade dos nossos resultados.

\section{CONCLUSÃO}

Este estudo sugere que existe grande disparidade de acesso e eficiência no manejo do CP no Brasil. Esta disparidade parece estar influenciada por fatores econômicos e demográficos. Foi possível ainda identificar alguns estados com grande ineficiência,

comprovando a falência do sistema público e universal de saúde.

FIGURAS

Figura 1. Percentual de cirurgias realizadas com intuito curativo para CP (Regiões em vermelho, Estados em azul, País em verde).

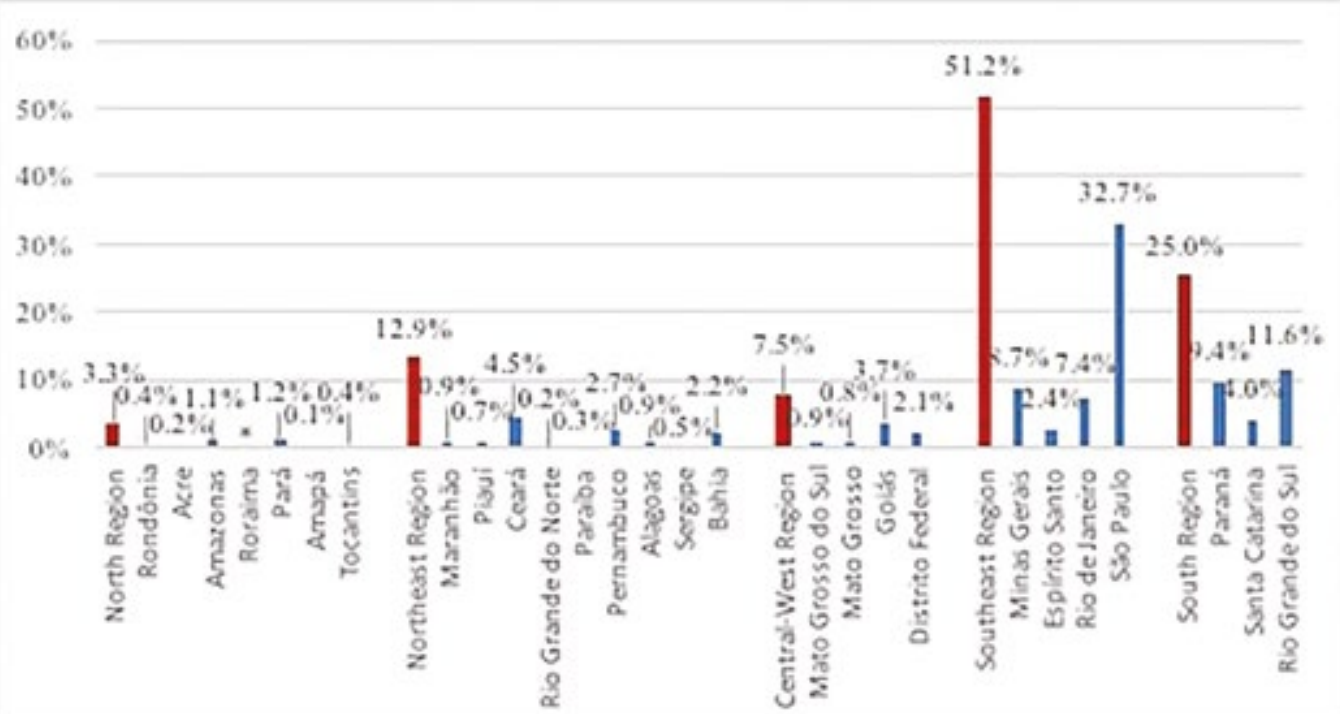

Figura 2. Mortalidade por CP por 100 mil habitantes/ano (Regiões em vermelho, Estados em azul, País em verde).

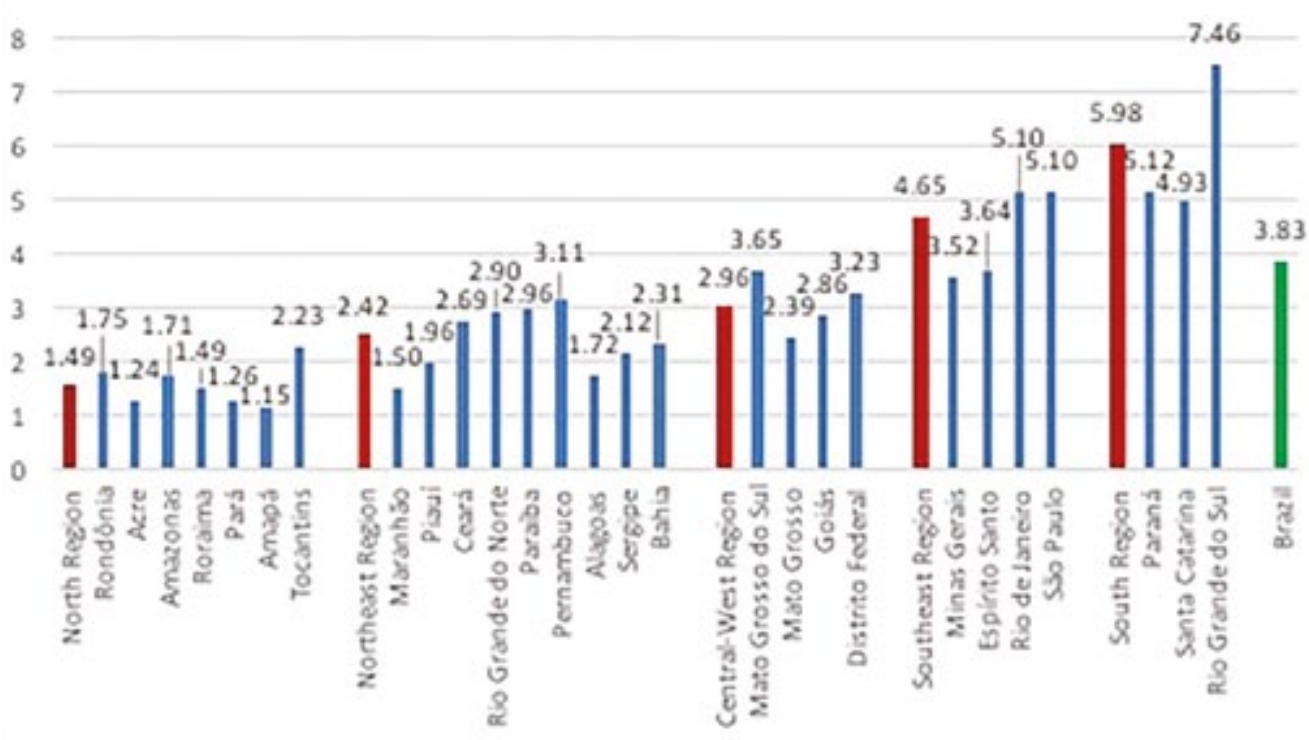


Figura 3. Taxa de mortalidade relacionada ao procedimento cirúrgico com intenção curativa (Regiões em vermeIho, Estados em azul, País em verde).

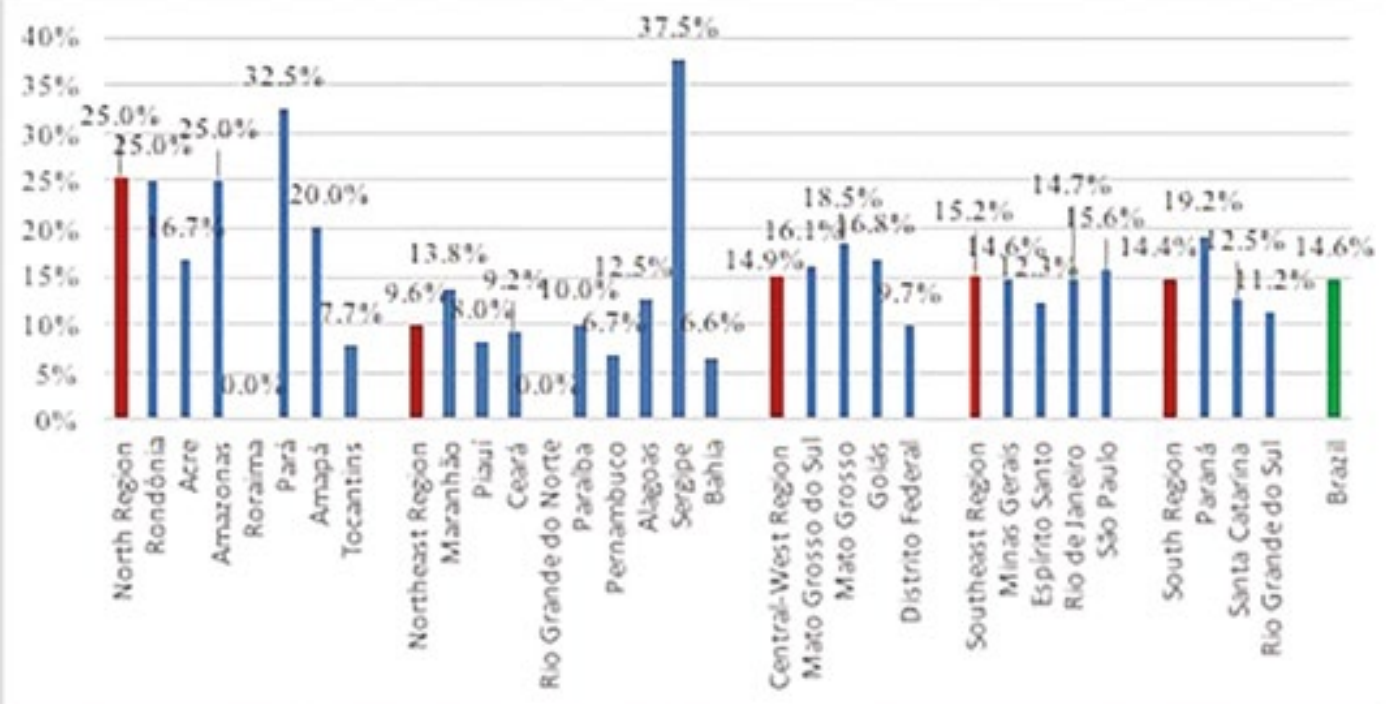

TABELAS

Tabela 1. Relação entre mortalidade específica por CP e número de procedimentos, PIB per capita e população.

\begin{tabular}{|c|c|c|}
\hline & $\begin{array}{l}\text { Univariate, coefficient }(95 \% \mathrm{Cl} \text {; } \\
\text { p-value) }\end{array}$ & $\begin{array}{l}\text { Multivariate, coefficient } \\
(95 \% \mathrm{Cl} \text {; p-value) }\end{array}$ \\
\hline $\begin{array}{l}\text { Number of } \\
\text { procedures }\end{array}$ & $0.24(0.12,0.36 ; p<0.001)$ & $\begin{array}{c}0.47(0.02,0.92 ; \\
p=0.04)\end{array}$ \\
\hline $\begin{array}{l}\text { GDP per } \\
\text { capita }\end{array}$ & $1.18(0.68,1.68 ; p<0.001)$ & $\begin{array}{c}0.72(0.06,1.38 ; \\
p=0.033)\end{array}$ \\
\hline Population & $0.57(0.18,0.95 ; \mathrm{p}=0.004)$ & $\begin{array}{l}-1.04(-2.24,0.17 \\
p=0.092)\end{array}$ \\
\hline
\end{tabular}

Tabela 2. Relação entre o período de permanência e o número de procedimentos, PIB per capita e população.

\begin{tabular}{|c|c|c|}
\hline & $\begin{array}{l}\text { Univariate, coefficient (95\% Cl; } \\
\text { p-value) }\end{array}$ & $\begin{array}{l}\text { Multivariate, coefficient } \\
\text { (95\% Cl; p-value) }\end{array}$ \\
\hline $\begin{array}{l}\text { Number of } \\
\text { procedures }\end{array}$ & $-0.01(-0.05,0.03 ; p=0.581)$ & $\begin{array}{c}0.04(-0.12,0.21 \\
p=0.593)\end{array}$ \\
\hline $\begin{array}{l}\text { GDP per } \\
\text { capita }\end{array}$ & $0.07(-0.11,0.24 ; \mathrm{p}=0.466)$ & $\begin{array}{l}0.12(-0.11,0.34 \\
\quad p=0.301)\end{array}$ \\
\hline Population & $-0.04(-0.14,0.06 ; p=0.414)$ & $\begin{array}{c}-0.2(-0.64,0.24 \\
p=0.376)\end{array}$ \\
\hline
\end{tabular}

\section{REFERÊNCIAS}

1. L. Parker, Sheryl, et al. Cancer Statistics, 1997. A Cancer Journal for Clinicians. Janeiro/Fevereiro , 1997, Vol. 47, 1.

2. R. Hamilton, Stanley and A. Aaltonen, Lauri.Pathology and Genetics of Tumours of the Digestive System. Lyon . IARC Library Cataloguing in Publication

3. J. Kneuertz, Peter, et al. Risk of Morbidity and Mortality Following Hepato-Pancreato-Biliary Surgery. Vou 16, astrointer

4. Lei 8080/90. Dispõe sobre as condições para a promoção, proteção e recuperação da saúde, a organização e ó funcionamento dos serviços correspondentes e dá outras providencias. Diârio Oricial da

5. L. dos Santos, Daniel and E. Gerhardt, Tatiana vientifica no contexlo do Sistema Unico de Saúde. Revista Gaúcha de Enfermagem. Março, 2008, Vol. 29, 1. 6. Ministerio da Saúde. Informaçôes de Saúde (TABNET). DATASUS. OUline] DATASUS - Departamento de Informálica do SUS. http.//WWW2.datasus.gov.br/

7. IBGE - Instituto Brasileiro de Geografia e Estatística. IBGE - Instituto Brasileiro de Geografia e Esta-

8. A. Makary, Martin, et al. Pancreaticoduodenec- Desigualdades sociais e saúde no Brasil: produção istica. [Online] S/ Data. http://www.ibge.gov.br/ tomy in the very elderly. Journal of Gastrointestinal Surgery. March, 2006, Vol. 10, 3.

9. D. Abeloff, Martin, et al. Abeloff's Clinical Oncology. Philadelphia : Churchill Livingstone; 4 edition 10.

10. C. T. Lagioia, Umbelina, et al. A gestão por processos gera melhoria de qualidade e redução de custos: o caso da unidade de ortopedia e traumatologia do hospital das clinicas da Universidade Federal de Pernambuco. Revista Contabildade \& Finanças. Setembro/Dezembro, vol.19 no.48 São Paulo Sept./Dec 2008, Vol. 19, n 48

1-Serviço de Cirurgia Oncológica do HSI

2- Georgia Institute of Technology, Atlanta, GA, US) 3- Grupo Oncoclínicas

4- IEP São Lucas, Universidade de Campinas

Endereço para correspondência:

marco le par 